\title{
Management of Breast Cancer in Visceral Surgery of CNHU-HKM of Cotonou in Benin
}

\author{
Dansou Gaspard Gbessi ${ }^{*}$, Ismail Lawani2 ${ }^{2}$, Chrystelle Tawo-Nounagnon 1 , \\ Francis Moïse Dossou'2, Yacoubou Imorou Souaïbou', Delphin Kuassi Mehinto3, \\ Jean-Léon Olory-Togbe', Kémoko Osséni Bagnan ${ }^{3}$, Nazaire Padonou ${ }^{1}$ \\ ${ }^{1}$ Visceral Surgery A CNHU-HKM, Cotonou, Benin \\ ${ }^{2}$ General Surgery CHDU-OP of Porto-Novo, Porto-Novo, Benin \\ ${ }^{3}$ Visceral Surgery B CNHU-HKM of Cotonou, Cotonou, Benin \\ Email: "gbessi1@yahoo.fr
}

Received 5 February 2016; accepted 22 March 2016; published 25 March 2016

Copyright (C) 2016 by authors and Scientific Research Publishing Inc.

This work is licensed under the Creative Commons Attribution International License (CC BY). http://creativecommons.org/licenses/by/4.0/

c) (i) Open Access

\begin{abstract}
Breast cancer affects more females than males. It occurs earlier with the black females and is often diagnosed at a stage of complication. This study aims to describe its epidemiological characteristics, the changing stages, and to analyze the treatment and means of interdisciplinary and international cooperation in its care and treatment. Our study was retrospective, descriptive and analytic on breast cancer patients received at CNHU-HKM of Cotonou between January 1, 2001 and December 31,2013 . Breast cancer accounts for $24.24 \%$ of mammary pathologies and most affects the left breast. Females aged from 40 to 59 years old are the most affected. For males, the average age was 47.5 years old. Patients mostly consult at a later stage where the tumor is already palpable with nodal metastasis. Breast echography and mammography had been done only for $23.85 \%$ of the cases. In an anatomical pathology perspective, the invasive ductal carcinoma was the most common type with $\mathbf{8 7 . 2 \%}$. For the therapeutical aspect, a radical mastectomy according to Patey was realized in $81.65 \%$ of the cases; $48.65 \%$ underwent a chemotherapy and $14.7 \%$ were able to do a radiotherapy. The chance at life for patients was decreasing while the life time is increasing. Also, the average life span of patients was increased by the combination of diverse types of treatments notably when radiotherapy is done. The incidence of breast cancers in Visceral Surgery A and B department of CNHU-HKM-Cotonou is increasing over years.
\end{abstract}

\section{Keywords}

Cancer, Breast, Epidemiology, Stage, Diagnosis, Chemotherapy, Radiotherapy

\footnotetext{
"Corresponding author.
}

How to cite this paper: Gbessi, D.G., Lawani, I., Tawo-Nounagnon, C., Dossou, F.M., Souaïbou, Y.I., Mehinto, D.K., OloryTogbe, J.-L., Bagnan, K.O. and Padonou, N. (2016) Management of Breast Cancer in Visceral Surgery of CNHU-HKM of Cotonou in Benin. Surgical Science, 7, 170-176. http://dx.doi.org/10.4236/ss.2016.73022 


\section{Introduction}

Breast cancer is an abnormal and uncontrolled cell of the mammary gland proliferation. It is a major problem of public health around the world. Breasts play an important role in the femininity and the body image of women. Several pathologies including cancer can affect them. In the world, in 2002, it was found near 1.1 million new cases with more than 400,000 deaths due to breast cancer. The incidence seems to increase regularly with over 1.6 million new cases in 2010 and 425,000 deaths [1]. In Europe the breast cancer is (men and women combined) with 13.3\% of incident cases behind colorectal cancer [2]. In France more than 50,000 new cases of breast cancer were diagnosed in 2010 with more than 11,000 deaths in the same year. In Africa, particularly in Benin, breast cancer is not uncommon. Diagnostic orientation is most often made by the couple ultrasound-mammography and confirmation is given by histological examination. Its support must be multidisciplinary: surgery, chemotherapy, radiotherapy, hormone therapy. Due to therapeutic advances, breast cancer mortality decreased since 1990s in developed countries; but it continued to grow in developing countries from the fact, above all, the lack of diagnostic and therapeutic [3]. Its efficient support is experiencing financial and geographical barriers. Faced with this situation, we proposed to the point of different therapeutic methods and their importance in the improvement of the survival of patients.

\section{Materials and Methods}

Our study took place in the University clinics of visceral surgery A and B of CNHU/HKM. It was retrospective, descriptive and analytical; held from $1^{\text {st }}$ January 2001 to 31 December 2013. We conducted a comprehensive census of patients with the breast cancer admitted clinical therein and during the study period. The sample was exhaustive type taking into account all cases of breast cancer in the study services according to the criteria of selection of the study. Were included in this study, patients who: suffering of a breast cancer confirmed by pathologic examination and supported in the framework and the study period; or had a tumor of the breast at a late stage with all the clinical characters of malignancy; or who had a complete medical record. Were excluded from this study, patients with benign tumors of the breast; patients whose medical records were incomplete.

The variables studied: Non-dependent variables: age, sex, history, histological type, year of hospitalization, clinical signs, paraclinical balance results. The dependent variables: selected diagnosis, treatment, stage scalable, duration of hospitalization, postoperative. To collect data, we proceeded in stages: as a first step, we have consulted the hospital registers of services of visceral surgery where we listed all cases of breast admitted during the study period. This has enabled us to raise numbers of records, diagnostics of entry and/or exit; as a second step, we searched records in the services of visceral surgery, internal medicine, and then to the archives of the National Hospital and University Hubert Koutoucou Maga (CNHU-HKM) Cotonou. During the search of the files, we got 109 cases of breast cancers that have met our inclusion criteria.

The anatomopathologic result which was not included in some cases sought to the anatomy pathology laboratory. We filled the recount form from each folder. In what keeps the survivors, conducted us three series of research, on the basis of data from the records of the patients (home address, phone number). The processing and analysis of data were made using Microsoft Word ${ }^{\circledR} 2007$ software, Microsoft Excel ${ }^{\circledR} 2007$ and SPSS. Analysis of the data has been possible thanks to chi2 test. The information obtained in this survey remained confidential.

\section{Results}

From $1^{\text {st }}$ January 2001 to 31 December 2013, we have listed 495 cases of mammary tumors; among these we identified histologically confirmed 109 cases of breast cancer, or a frequency of $24.24 \%$. In our study, $98.2 \%$ of patients were women compared with $1.8 \%$ were men.

The distribution of the 109 patients presented itself as indicated in Table 1.

We note 72 cases $(66.1 \%)$ of malignant tumours between 40 and 59 years with a maximum of frequency in the 45 to 49 age group $(21.1 \%)$.

The average age was 48 years with extremes of 25 and 73 years (Table 2).

Breast cancer was more found in the paucipares and the multiparous in 71 cases (54.21\%).Female patient, 59 cases (55.14\%) were postmenopausal before age 50. Mammary pathology personal history was found in 15 patients (13.8\%), and 99 patients (90.83\%) had no family pathological history. 
Table 1. Distribution of patients according to the age group.

\begin{tabular}{cccc}
\hline Age group (years) & Staff & Percentage (\%) \\
\hline$[25-29]$ & 3 & 2.8 \\
{$[30-34]$} & 6 & 5.5 \\
{$[35-39]$} & 12 & 11 \\
{$[40-44]$} & 17 & 15.6 \\
{$[45-49]$} & 23 & 21.1 \\
{$[50-54]$} & 17 & 15.6 \\
{$[55-59]$} & 15 & 13.8 \\
{$[60-64]$} & 9 & 8.3 \\
{$[65-69]$} & 4 & 3.7 \\
{$[70-74]$} & 3 & 2.8 \\
Total & $\mathbf{1 0 9}$ & $\mathbf{1 0 0}$ \\
\hline
\end{tabular}

Table 2. Distribution of patients at parity.

\begin{tabular}{ccc}
\hline Parity & Workforce & Percentage (\%) \\
Nulliparous & 3 & 2.8 \\
First-calf heifers & 7 & 6.54 \\
Paucipares & 36 & 22.43 \\
Multiparous & 35 & 31.78 \\
Large multiparous & 11 & 20.56 \\
Total & $\mathbf{1 0 7}$ & $\mathbf{1 0 0}$ \\
\hline
\end{tabular}

\subsection{Clinical Study}

Clinically, the most common reason for consultation of the disease is the fortuitous discovery of a swelling or nodule at the level of the breast in 75 cases (68.8\%) (Table 3).

The majority of patients had consulted within a period of less than 12 months (2 weeks to 11 months)

The characteristics and classification of tumor are reflected in Table 4 and Table 5.

The majority of patients had consulted when the tumor is large.

39 (35.78\%) patients had accessed at stage II, and 43 patients (39.45\%) in stage III; thus consultant stages II and III patients accounted for most of three quarters, $75.23 \%$ of the study population. In our study, 94 (86.26\%) patients had lymph nodes axillary and sus-clavicular which 11 patients (10.09\%) metastases to other organs. On the paraclinical plan, mammography and breast ultrasound were performed in 26 patients (23.85\%). 39 (35.78\%) patients had accessed at stage II, and 43 patients (39.45\%) in stage III; thus patients of consultant stages II and III accounted for most of three quarters, $75.23 \%$ of the study population (Table 5).

\subsection{Tumor Varieties}

Invasive ductal carcinoma dominates breast cancer pathology (Table 6).

The histological grade dominant Scarf Bloom and Richardson (SBR) consists of tumors to the second histological grade (SBR II) with 77 cases (81.05\%).

Therapeutically, surgery, chemotherapy, radiation therapy and hormone therapy are the means used in the management of patients with breast cancer.

In our study, radical surgical treatment according to the technique of Patey had been practiced in 90 patients (82.57\%). Conservative surgical treatment was practiced in 11 patients (7.3\%), clean 3 patients surgery, nonsurgical treatment in 3 cases. The treatment of two patients had not been specified. 
Table 3. Reasons for consultation.

\begin{tabular}{ccc}
\hline Reasons for consultation & Staff & Percentage (\%) \\
\hline Nodule & 75 & 68.8 \\
Painful phenomena & 18 & 16.5 \\
Axillary lymphadenopathy & 7 & 6.4 \\
Ulceration & 5 & 4.6 \\
Flow nipple & 3 & 2.8 \\
Pleural effusion & 1 & 0.9 \\
Total & $\mathbf{1 0 9}$ & $\mathbf{1 0 0}$ \\
\hline
\end{tabular}

Table 4. Tumor size.

\begin{tabular}{ccc}
\hline Size of the tumor $(\mathbf{c m})$ & Workforce & Percentage (\%) \\
\hline $\mathrm{T} 0$ & 0 & 0 \\
$\mathrm{~T} 1(\mathrm{~T}<2)$ & 3 & 2.75 \\
$\mathrm{~T} 2(2<\mathrm{T}<5)$ & 54 & 49.54 \\
$\mathrm{~T} 3(\mathrm{~T}>5)$ & 40 & 36.7 \\
$\mathrm{~T} 4$ & 12 & 11.01 \\
Total & $\mathbf{1 0 9}$ & $\mathbf{1 0 0}$ \\
\hline
\end{tabular}

Table 5. Clinical stages of tumor.

\begin{tabular}{ccc}
\hline Stages & staff & Percentage (\%) \\
Stage I & 15 & 13.76 \\
Stage II & 39 & 35.78 \\
Stage III & 43 & 39.45 \\
Stage IV & 12 & 11.01 \\
Total & $\mathbf{1 0 9}$ & $\mathbf{1 0 0}$ \\
\hline
\end{tabular}

Table 6. Tumor varieties.

\begin{tabular}{ccc}
\hline Varieties & Workforce & Percentage (\%) \\
\hline Infiltrating ductal carcinoma & 95 & 87.2 \\
Ductal carcinoma in situ & 5 & 4.7 \\
Mucosecretant ductal carcinoma & 2 & 1.8 \\
Inflammatory Carcinoma & 1 & 0.9 \\
Ductal carcinoma Medullary & 1 & 0.9 \\
Papillary ductal carcinoma & 2 & 1.8 \\
Ductal carcinoma lobular in situ & 1 & 0.9 \\
Adenocarcinoma, unspecified & 1 & 0.9 \\
Adenocarcinoma Signet Ring & 1 & 0.9 \\
Total & $\mathbf{1 0 9}$ & $\mathbf{1 0 0}$ \\
\hline
\end{tabular}


Chemotherapy had been achieved in 54 patients (48.62\%). The most used protocols were: 5-fluorouracil, Adriamicine and Cyclophosphamide (FAC) in 24 patients (52.2\%), Cyclophosphamide (Endoxan ${ }^{\circledR}$ ) monotherapy in 13 patients (28.2\%).

Radiotherapy was not available in Benin. 16 patients had been able to travel abroad to qualify: 12 patients in Ghana, 1 patient in Tunisia, and 3 patients in Paris. Hormone therapy had been practiced at 8 post-menopausal patients on 107 female patients.

In evolutionary terms, patients receiving no treatment have a lifespan of between 0 and 5 months; patients who received treatment as surgery + chemotherapy were between 10 and 20 months, better life to those who have only had one and lesser surgery compared with those who had radiotherapy. Better support was the association surgery + chemotherapy and radiotherapy.

\section{Discussion}

Support for malignant tumors of the breast in the University clinics of Cotonou in teaching hospital CNHU-HKM, knows few limits in comprehensive treatment of this condition, due to chemotherapy not practised in all patients and limits exist in Benin of radiotherapy.

Epidemiologically, we counted 109 cases of breast cancer, a $24.24 \%$ frequency. The male rate was $1.8 \%$ with a female (98.2\%). Our results are going in the same direction as those found among males in the literature: in the United States reported 0.7\% [4], in Tunisia 1\% [5], in Gabon and Nigeria 3\% to 4.16\%, respectively [6] [7].

In our study, the average age of women was 48 years. This result is similar to 46 years found in the U.S. [8]. However in Caucasian women, the average age was 67 years. In our study it was 47.5 years. Other authors in Nigeria, reported among men, an average age between 57.9 years and 65 years [5] [7] [9]. We conclude then at a relatively young age of patients in our study as well in men than in women.

The multiparity and the pauciparity found in $66 \%$ of our patients have been noted in $75 \%$ and $80 \%$ by some authors of literature. Menopausal women in our study accounted for 70\%. In the literature, studies founded [10]. The concept of family history of breast cancer founded 6\% of cases. Authors in Tunisia noted 14\% of patients with this type of history [11]. 14\% of patients have a personal history of finally in our study. Patients in our study have consulted for a nodule or swelling of the breast. However in the European series, screening was done for subclinical lesions.

The consultation period is between 2 weeks and 12 months is 63 per cent of cases. Studies reported $59 \%$ of patients [12]. Tumor sizes ranged between $2 \mathrm{~cm}$ and $5 \mathrm{~cm}$ or greater than $5 \mathrm{~cm}$ with a percentage of $75.23 \%$ because patients didn't came to hospital early. Our results are the same as those found in African literature, 72.8\% [12] [13]. In Europe, the tumor size is less than $2 \mathrm{~cm}$ and $80 \%$ of patients were T1 and T2 [14] [15]. Patients in our study have consulted at stage II or III is 62 per cent. In developed countries, consultations were made in the early stages [16]. This delay in the consultation in our regions of Africa is multifactorial: problem of shame, fear of the diagnosis, haunting of mastectomy but also the absence of mass screening [16].

On the paraclinical plan, only 23.85\% could make breast ultrasound associated with mammography due to the limited financial means to patients. The anatomopathologic examination found $87.2 \%$ of invasive ductal carcinoma and lymph node invasion in $68 \%$ of our patients. Our results are similar to those those authors who had respectively $90.5 \%$; $88.25 \%$ and $81 \%$ of ductal carcinoma infiltrating [17]-[19].

Therapeutically, in our study, 81.65\% benefited from a radical surgery according to Patey. [20] in 2010 reported $65.71 \%$ radical cure. [21] have reported similar results, 61\%. Conservative treatment was directed in $7.3 \%$ of cases. [20] in 2010 reported that $10 \%$ of patients have benefited from conservative surgery. We had 5 cases of carcinoma in situ treated in the following way: 2 cases of radical cure according to Patey only; 2 cases of conservative surgery alone, 1 case of radical surgery with chemotherapy. According to the recommendations of the high authority of health in France support this tumor variety was either a radical therapy combined with radiotherapy if the technique of Sentinel lymph node is positive or a conservative treatment in the case of negative sentinel lymph node [21].

In our study, 48.62\% had chemotherapy. Our results differ from those others who have found 38\% [17]. 5fluorouracil, Adriamicine and Cyclophosphamide (FAC) Protocol was the most used 52.2\%. The high authority of health in France offers a combination of Anthracyclines and Taxanes as Protocol [21]. Radiation therapy was 14\%. In our case patients were sent to Ghana, Tunisia or France. [22] in Gabon reported 14\% of patients who have radiation therapy study. Our results differ from those of [17] who reported 63\% Many African countries including 
Benin have not a technical platform for the realization of the radiotherapy. With regard to hormone therapy, only a patient on 9 could benefit research of hormone receptors prior to the institution of the treatment and this was done in France. The rate of living patients after 5 years was $10 \%$ and at 10 years was 3\%. In African countries in general, this rate was 57 per cent after a decline of 5 years. More patients having radiotherapy treatment have a greater than other life. This demonstrates the importance of radiotherapy in the management of malignant tumors of the breast.

\section{Conclusion}

Breast cancer is a serious and fatal disease which affects both women and men but with a high frequency among women and at a relatively young age in our country. In our African countries including Benin, its diagnosis is late with a difficult support thereby causing high mortality. Mass screening could help to significantly reduce this frequency in our African countries but the difficulties of this activity are financial.

\section{References}

[1] Forouzan, M.H., Foreman, K.J. and Delossantos, A.M. (2011) Breast and Cervical Cancer in 187 Countries between 1980 and 2010: A Systematic Analysis. The Lancet, 378, 1461-1484.

[2] Boyle, P. (2005) Cancer Incidence and Mortality in Europe, 2004. Annals of Oncology, 16, 481-488. http://dx.doi.org/10.1093/annonc/mdi098

[3] Rey, J.-L. (2008) How to Prevent Cancers in Developing Countries? Medicine Review, 38, 5.

[4] Jemal, A., Murray, T. and Ward, E. (2005) Cancer statistics, 2005. CA: A Cancer Journal for Clinicians, 55, 10-30. http://dx.doi.org/10.3322/canjclin.55.1.10

[5] Dhiab, T.B., Bouzid, T., Gamoudi, A., Hassouna, J.B., Khomsi, F., Boussen, H., Benna, F., El May, A., Hechiche, M. and Rahal, K. (2005) Human Breast Cancer: About 123 Cases Collected at the Institute Salah Azaiz of Tunis from 1979 to 1999. Bulletin du Cancer, 92, 281-285.

[6] Mervie, L.P., Jouvance, O. and Naepels, P. (2011) Does It the Risk’s Factors of Breast Cancer? Gynecology Obstetrics and Fertility A, 39, 486-490.

[7] Kidmas, A.T., Ugwu, B.T., Manasseh, A.N., Iya, D. and Opaluwa, A.S. (2005) Male Breast Malignancy in Jos University Teaching Hospital. West African Journal of Medicine, 24, 36-40. http://dx.doi.org/10.4314/wajm.v24i1.28160

[8] Bowen, R.L., Duffy, S.W., Ryan, D.A., Hart, .I.R. and Jones, J.L. (2008) Early Onset of Breast Cancer in a Group of British Black Women. British Journal of Cancer, 98, 277-281. http://dx.doi.org/10.1038/sj.bjc.6604174

[9] Sano, D., Dao, B., Lankoandé, J., Touré, B., Sakandé, B., Traoré, B., Traoré, S.S., Wandaogo, A., Dakouré, R. and Sanou, A. (1977) Cancer Man in the African Environment. About 5 Cases Seen at the Hospital-University of Ouagadougou (Burkina-Faso) Centre. Bulletin Cancer, 84, 175-177.

[10] Odigie, V.I., Yusufu, L.M., Rafindadi, A. and Darocha-Afodu, J.T. (2003) Breast Cancer in Zaria. Nigerian Journal Surgery, 9, 46-50.

[11] Ben, A.S., Aloulou, S., Bibi, M., Landolsi, A. and Kallel, L. (2002) Breast Cancer Prognosis in Tunisian Women: Analis of Hospital Series of 729 Patients. Public Health, 3, 231-241.

[12] Sarre, B., Ogougbemy, M., Dotou, C., Lopy, J., Ndiann, Frank, K. and Diouf, M.B. (2006) Epidemiogical, Therapeutic and Prognosis Aspects of Breast Cancer about 473 Cases Collected in Principal Hospital of Dakar. Dakar Medical, 51, 92-96.

[13] Kene, T.S., Odigie, V.I., Yusufu, L.M.D., Yusuf, B.O., Shehu, S.M. and Kase, S.M. (2010) Pattern of Presentation and Survival of Breast Cancer in Teaching Hospital in North Western Nigeria. OMJ, 25, 104-107. http://dx.doi.org/10.5001/omj.2010.29

[14] Marty, M., Rossignol, C. and Srruys, G. (1992) The Breast Cancer Diagnosis, National Study of the CANAM: Analysis of 307 Cases. La Presse Médicale, 21, 1017-1022.

[15] Salmon, R.J. (1998) Evolution of Breast Cancer Surgery. Bulletin du Cancer, 88, 539-543.

[16] Piccart, M., Dochy, E. and Cardosso, F. (2003) Therapeutic News in the Medical Treatment of Breast Cancer. Bulletin du Cancer, 90, 46-52.

[17] Ganry, O. (1997) Cancers of the Breast in the Somme in 1990: Descriptive Epidemiology, Treatment, and Survival. Revue Francaise de Gynécologie et d'Obstétrique, 926, 382-388.

[18] Demange, L. (1992) Limited Lumpectomy Combined with Peroperative Curietherapy for Conservative Treatment of Breast Cancer. Journal Gynecology Obstetric Biologic Reproduction, 21, 479-483. 
[19] Uzan, S. (1998) Breast Cancer. Epidemiology, Anatomy Pathology, Screening, Diagnosis, Evolution, Principle of Treatment. The Journal of the Practitioner, 48.

[20] Togo, A., Traoré, A. and Traoré, C. (2010) Breast Cancer in Bamako Hospitals. Diagnosis and Treatment. African Journal of Cancer A, 2, 88-91. http://dx.doi.org/10.1007/s12558-010-0060-x

[21] (2010) High Authority in Health. Therapeutic Support. In: Guide ALD-Breast Cancer, 15-27.

[22] Mayi-Tsonga, S., Belembaogo, E. and Meye J.F. (2009) The Cancers of the Breast in Gabon: Epidemiological, Diagnostic, and Therapeutic Aspects. African Journal of Cancer, 1, 11-15. 